\title{
HOMOLOGY OF ODOMETERS
}

\author{
EDUARDO SCARPARO
}

\begin{abstract}
We compute the homology groups of transformation groupoids associated with odometers and show that certain $\left(\mathbb{Z} \rtimes \mathbb{Z}_{2}\right)$-odometers give rise to counterexamples to the HK conjecture, which relates the homology of an essentially principal, minimal, ample groupoid $G$ with the K-theory of $C_{r}^{*}(G)$. We also show that transformation grupoids of odometers satisfy the AH conjecture.
\end{abstract}

\section{INTRODUCTION}

Given a decreasing sequence $\left(\Gamma_{i}\right)_{i \in \mathbb{N}}$ of finite index subgroups of a group $\Gamma$, there is an action of $\Gamma$ on $\lim \Gamma / \Gamma_{i}$ given by left-multiplication. This action is called an odometer, and it has been extensively studied.

For example, in [18, Orfanos obtained several properties of crossed products associated to odometers, in the case that the acting group is amenable. In [12, Ioana studied these actions in the measurable setting, showing orbit equivalence superrigidity when the acting group has property $(\mathrm{T})$, and, in [6], Cortez and Medynets studied continuous orbit equivalence and topological full groups of odometers.

In [15, Matui formulated two conjectures about homology groups and K-theory of second countable, étale, essentially principal, minimal groupoids with unit space homeomorphic to the Cantor set. The first one (HK conjecture) predicts that, given such a groupoid $G$, the following holds:

$$
K_{*}\left(C_{r}^{*}(G)\right) \simeq \bigoplus_{k \geq 0} H_{2 k+*}(G) \text { for } *=0,1 .
$$

The HK conjecture has been verified for several classes of groupoids (see [19] and [10] for recent developments).

The second conjecture (AH conjecture) relates the abelianization of the topological full group of $G$ with the two first homology groups of $G$. It has been verified for principal, almost finite groupoids, and groupoids arising from products of one-sided shifts of finite type ([15).

In this note, we show that certain $\left(\mathbb{Z} \rtimes \mathbb{Z}_{2}\right)$-odometers are counterexamples to the HK conjecture. The particular class of $\left(\mathbb{Z} \rtimes \mathbb{Z}_{2}\right)$-odometers that we consider has already appeared elsewhere (e.g., in [17, Section 2.4], 9, Example 7.5] and [20, Example 4.3]). Moreover, in [1, 10.11.5(c)], using a different picture, Blackadar computed the K-theory of the crossed products associated to these odometers, and, in [13], Kumjian showed that these crossed products are AF (approximately finitedimensional).

We also show that the AH conjecture holds for transformation groupoids associated to odometers.

The author was supported by CNPq, Brazil, 167983/2017-2. 
The paper is organized as follows. In Section 2, we collect basic facts about odometers, and characterize when an odometer is topologically free. We also describe the K-theory and homology of odometers, and we recall the the definitions of ample groupoids and their homology groups.

In Section 3, we compute the K-theory and homology of certain $\left(\mathbb{Z} \rtimes \mathbb{Z}_{2}\right)$ odometers and show that they are counterexamples to the HK conjecture. In Section 4. we show that transformation groupoids of odometers satisfy the AH conjecture.

Acknowledgement. The author thanks Kevin Aguyar Brix for inspiring conversations about homology of groupoids.

\section{Preliminaries}

2.1. Odometers. Let $\Gamma$ be a group and $\left(\Gamma_{i}\right)_{i \in \mathbb{N}}$ a sequence of finite index subgroups of $\Gamma$ such that, for every $i \in \mathbb{N}, \Gamma_{i} \geqslant \Gamma_{i+1}$.

For each $i \in \mathbb{N}$, let $p_{i}: \Gamma / \Gamma_{i+1} \rightarrow \Gamma / \Gamma_{i}$ be the surjection given by

$$
p_{i}\left(\gamma \Gamma_{i+1}\right):=\gamma \Gamma_{i} \text {, for } \gamma \in \Gamma .
$$

Let $X:=\lim \left(\Gamma / \Gamma_{i}, p_{i}\right)=\left\{\left(x_{i}\right) \in \Pi \Gamma / \Gamma_{i}: p_{i}\left(x_{i+1}\right)=x_{i}, \forall i \in \mathbb{N}\right\}$. Then $X$ is homeomorphic to the Cantor set and $\Gamma$ acts in a minimal way on $X$ by $\gamma\left(x_{i}\right):=$ $\left(\gamma x_{i}\right)$, for $\gamma \in \Gamma$ and $\left(x_{i}\right) \in X$. This action is called an odometer (terminology from [6]). See [8, Fait 2.1.4] and [9, Proposition A.1] for more abstract characterizations of odometers.

Note that, if $\left(x_{i}\right),\left(y_{i}\right) \in X$, and there exists $i_{0}$ such that $x_{i_{0}}=y_{i_{0}}$, then, for $1 \leq i \leq i_{0}$, it holds that $x_{i}=y_{i}$.

Given $j \geq 1$ and $g \Gamma_{j} \in \Gamma / \Gamma_{j}$, let $U\left(j, g \Gamma_{j}\right):=\left\{\left(x_{i}\right) \in X: x_{j}=g \Gamma_{j}\right\}$. Then $\left\{U\left(j, g \Gamma_{j}\right): j \in \mathbb{N}, g \Gamma_{j} \in \Gamma / \Gamma_{j}\right\}$ is a basis for $X$ consisting of compact-open sets.

Recall that an action of a group $\Gamma$ on a locally compact Hausdorff space $Y$ is said to be topologically free if, for each $\gamma \in \Gamma \backslash\{e\}$, the set of points of $Y$ fixed by $\gamma$ has empty interior.

Proposition 2.1. An odometer $\Gamma \curvearrowright X:=\lim \Gamma / \Gamma_{i}$ is topologically free if and only if, for every $\gamma \in \cap \Gamma_{i} \backslash\{e\}$ and $j \geq 1$, there exists $b \in \Gamma_{j}$ such that $b^{-1} \gamma b \notin \cap \Gamma_{i}$.

Proof. Suppose that the $\Gamma$-action is not topologically free. This implies that there exists $\gamma^{\prime} \in \Gamma \backslash\{e\}$ and $V \subset X$ non-empty and open such that each $x \in V$ is fixed by $\gamma^{\prime}$. Since the family $\left\{U\left(j, g \Gamma_{j}\right): j \in \mathbb{N}, g \Gamma_{j} \in \Gamma / \Gamma_{j}\right\}$ is an open basis for $X$, there exists $j \in \mathbb{N}$ and $g \Gamma_{j} \in \Gamma / \Gamma_{j}$ such that $U\left(j, g \Gamma_{j}\right)$ is fixed pointwise by $\gamma^{\prime}$. Since $U\left(j, g \Gamma_{j}\right)=g U\left(j, \Gamma_{j}\right)$, it follows that $\gamma:=g^{-1} \gamma^{\prime} g$ fixes pointwise $U\left(j, \Gamma_{j}\right)$.

Given $b \in \Gamma_{j}$, we have $\left(b \Gamma_{i}\right)_{i \in \mathbb{N}} \in U\left(j, \Gamma_{j}\right)$, hence $\left(\gamma b \Gamma_{i}\right)=\left(b \Gamma_{i}\right)$. Therefore, $b^{-1} \gamma b \in \cap \Gamma_{i}$. This concludes the backwards implication.

For the converse, assume that there exists $\gamma \in \cap \Gamma_{i} \backslash\{e\}$ and $j \geq 1$ such that, for every $b \in \Gamma_{j}, b^{-1} \gamma b \in \cap \Gamma_{i}$.

Given $\left(g_{i} \Gamma_{i}\right) \in U\left(j, \Gamma_{j}\right)$, by the definition of $X$, we have that, for $i \geq j, g_{i} \in \Gamma_{j}$. Therefore, for $i \geq j$, it holds that $\gamma g_{i} \Gamma_{i}=g_{i} \Gamma_{i}$, and this implies that $\gamma g_{i} \Gamma_{i}=g_{i} \Gamma_{i}$ for every $i$.

Hence, $\gamma\left(g_{i} \Gamma_{i}\right)=\left(g_{i} \Gamma_{i}\right)$ for any $\left(g_{i} \Gamma_{i}\right) \in U\left(j, \Gamma_{j}\right)$, and the action is not topologically free.

Example 2.2. Recall that the infinite dihedral group is the semidirect product $\mathbb{Z} \rtimes \mathbb{Z}_{2}$ associated to the action of $\mathbb{Z}_{2}$ on $\mathbb{Z}$ by multiplication by -1 . 
Let $\left(n_{i}\right)$ be a strictly increasing sequence of natural numbers such that $n_{i} \mid n_{i+1}$, for every $i \in \mathbb{N}$. Define $\Gamma:=\mathbb{Z} \rtimes \mathbb{Z}_{2}$ and, for $i \geq 1, \Gamma_{i}:=n_{i} \mathbb{Z} \rtimes \mathbb{Z}_{2}$. Then $\cap \Gamma_{i}=\{(0,0),(0,1)\}$. Moreover, for $j \geq 1$, we have that $\left(n_{j}, 0\right)(0,1)\left(-n_{j}, 0\right)=$ $\left(2 n_{j}, 1\right) \notin \cap \Gamma_{i}$. Therefore, $\Gamma \curvearrowright \lim \Gamma / \Gamma_{i}$ is topologically free.

Given $\Gamma \curvearrowright \lim \Gamma / \Gamma_{i}$ an odometer such that $\Gamma_{i} \unlhd \Gamma$ for every $i$, the $\Gamma$-action is free if and only if $\cap \Gamma_{i}=\{e\}$ (see [7, Section 2.1]). Furthermore, if the $\Gamma$-action is topologically free, then Proposition 2.1 implies that $\cap \Gamma_{i}=\{e\}$.

Recall that an action of a group $\Gamma$ on a set $X$ is said to be faithful if, for every $\gamma \in \Gamma \backslash\{e\}$, there exists $x \in X$ such that $\gamma x \neq x$. The group $\Gamma$ is said to be residually finite if, for every $\gamma \in \Gamma \backslash\{e\}$, there exists a finite group $F$ and a homomorphism $\varphi: \Gamma \rightarrow F$ such that $\varphi(\gamma) \neq e$.

In case there exists a faithful $\Gamma$-odometer $\Gamma \curvearrowright \lim \Gamma / \Gamma_{i}$, then, given $\gamma \in \Gamma \backslash$ $\{e\}$, there is $j \geq 1$ such that $\gamma$ acts non-trivially on $\Gamma / \Gamma_{j}$. In particular, $\Gamma$ is residually finite (this remark is from [4]). Conversely, if $\Gamma$ is countably infinite and residually finite, there exists a strictly decreasing sequence $\left(\Gamma_{i}\right)$ of finite index normal subgroups of $\Gamma$ such that $\cap \Gamma_{i}=\{e\}$, and the odometer $\Gamma \curvearrowright \lim \Gamma / \Gamma_{i}$ is free.

2.2. K-theory of odometers. Given a group $\Gamma$ acting on a compact Hausdorff space $X$, we will denote the canonical copy of $\Gamma$ in either $C(X) \rtimes_{r} \Gamma$ or $C(X) \rtimes \Gamma$ by $\left(\delta_{g}\right)_{g \in \Gamma}$.

The next result is an easy consequence of [11, Corollary 2.10].

Proposition 2.3. Let $\Lambda$ be a finite index subgroup of a group $\Gamma$. Then

$$
C(\Gamma / \Lambda) \rtimes_{r} \Gamma \simeq M_{\Gamma / \Lambda}(\mathbb{C}) \otimes C_{r}^{*}(\Lambda) .
$$

Proof. Take representatives $g_{1}, \ldots, g_{n}$ for $\Gamma / \Lambda$. For $1 \leq i \leq n$ and $g \in \Gamma$, let $h(i, g) \in \Lambda$ and $\sigma_{g}(i) \in\{1, \ldots, n\}$ be such that

$$
g g_{i}=g_{\sigma_{g}(i)} h(i, g) .
$$

Then there is a surjective $*$-homomorphism $\psi: C(\Gamma / \Lambda) \rtimes \Gamma \rightarrow M_{\Gamma / \Lambda}(\mathbb{C}) \otimes C_{r}^{*}(\Lambda)$ such that

$$
\psi\left(1_{\{l\}}\right)=e_{l, l} \otimes 1 \text { and } \psi\left(\delta_{g}\right)=\sum e_{\sigma_{g}(i), i} \otimes \delta_{h(i, g)}, \text { for } l \in \Gamma / \Lambda \text { and } g \in \Gamma .
$$

Let $\tau$ be the canonical faithful tracial state on $C_{r}^{*}(\Lambda)$ and $\varphi: M_{\Gamma / \Lambda}(\mathbb{C}) \rightarrow C(\Gamma / \Lambda)$ be the canonical conditional expectation. Then $\varphi \otimes \tau: M_{\Gamma / \Lambda}(\mathbb{C}) \otimes C_{r}^{*}(\Lambda) \rightarrow C(\Gamma / \Lambda)$ is a faithful conditional expectation such that $(\varphi \otimes \tau) \circ \psi$ is the canonical conditional expectation from $C(\Gamma / \Lambda) \rtimes \Gamma$ onto $C(\Gamma / \Lambda)$. In particular, $\psi$ factors through an isomorphism from $C(\Gamma / \Lambda) \rtimes_{r} \Gamma$ into $M_{\Gamma / \Lambda}(\mathbb{C}) \otimes C_{r}^{*}(\Lambda)$.

Hence, given an odometer $\Gamma \curvearrowright X=\lim \Gamma / \Gamma_{i}$, we have that $C(X) \rtimes_{r} \Gamma \simeq$ $\lim _{\longrightarrow} C\left(\Gamma / \Gamma_{i}\right) \rtimes_{r} \Gamma \simeq \lim _{\longrightarrow} M_{\Gamma / \Gamma_{i}}(\mathbb{C}) \otimes C_{r}^{*}\left(\Gamma_{i}\right)$ (this was observed already in [5] $)$. There$\overrightarrow{\text { fore, }}$

$$
K_{*}\left(C(X) \rtimes_{r} \Gamma\right) \simeq \lim _{\longrightarrow} K_{*}\left(C_{r}^{*}\left(\Gamma_{i}\right)\right)
$$


2.3. Homology of odometers. Given a group $\Gamma$, we denote by $\Gamma^{\prime}$ its commutator subgroup. Recall that $\Gamma_{\mathrm{ab}}:=\frac{\Gamma}{\Gamma^{\prime}}$ is the abelianization of $\Gamma$.

Let $\Lambda$ be a finite index subgroup of a group $\Gamma$. Let us recall the definition of the transfer map $\operatorname{tr}_{\Lambda}^{\Gamma}: H_{*}(\Gamma) \rightarrow H_{*}(\Lambda)$ (see, e.g., [3, Section III.9]).

Consider $\mathbb{Z}$ as a trivial $\Lambda$-module and $\mathbb{Z}^{\Gamma / \Lambda}$ as a permutation $\Gamma$-module. Let $i: \Lambda \rightarrow \Gamma$ be the inclusion and $\varphi: \mathbb{Z} \rightarrow \mathbb{Z}^{\Gamma / \Lambda}$ be the additive map given by $\varphi(1):=$ $\delta_{\Lambda}$. Then functoriality of $H_{*}$ gives a homomorphism $(i, \varphi)_{*}: H_{*}(\Lambda) \rightarrow H_{*}\left(\Gamma, \mathbb{Z}^{\Gamma / \Lambda}\right)$, which, by Shapiro's lemma, is an isomorphism.

Also let $\psi: \mathbb{Z} \rightarrow \mathbb{Z}^{\Gamma / \Lambda}$ be the additive map given by

$$
\psi(1):=\sum_{x \in \Gamma / \Lambda} \delta_{x}
$$

Note that $\psi$ is $\Gamma$-equivariant, hence it induces a homomorphism $H_{*}(\psi): H_{*}(\Gamma) \rightarrow$ $H_{*}\left(\Gamma, \mathbb{Z}^{\Gamma / \Lambda}\right)$. Define $\operatorname{tr}_{\Lambda}^{\Gamma}:=(i, \varphi)_{*}^{-1} \circ H_{*}(\psi): H_{*}(\Gamma) \rightarrow H_{*}(\Lambda)$.

For $*=0, \operatorname{tr}_{\Lambda}^{\Gamma}: \mathbb{Z} \rightarrow \mathbb{Z}$ is multiplication by $[\Gamma: \Lambda]$.

Take representatives $g_{1}, \ldots, g_{n}$ for $\Gamma / \Lambda$. For $1 \leq i \leq n$ and $g \in \Gamma$, let $h(i, g) \in \Lambda$ be such that $g g_{i}=g_{\sigma_{g}(i)} h(i, g)$ for some $\sigma_{g}(i) \in\{1, \ldots, n\}$. Then, for $*=1$, $\operatorname{tr}_{\Lambda}^{\Gamma}: \Gamma_{\mathrm{ab}} \rightarrow \Lambda_{\mathrm{ab}}$ is given by

$$
\operatorname{tr}_{\Lambda}^{\Gamma}\left(g \Gamma^{\prime}\right)=\sum_{i=1}^{n} h(i, g) \Lambda^{\prime}
$$

Proposition 2.4. Let $\Gamma \curvearrowright X=\lim \Gamma / \Gamma_{i}$ be an odometer. Then

$$
H_{*}(\Gamma, C(X, \mathbb{Z})) \simeq \underline{\lim }\left(H_{*}\left(\Gamma_{i}\right), \operatorname{tr}_{\Gamma_{i+1}}^{\Gamma_{i}}\right) .
$$

Proof. For $i \geq 1$, let $p_{i}: \Gamma / \Gamma_{i+1} \rightarrow \Gamma / \Gamma_{i}$ be as in (11) and $q_{i}: \mathbb{Z}^{\Gamma / \Gamma_{i}} \rightarrow \mathbb{Z}^{\Gamma / \Gamma_{i+1}}$ be given by $q_{i}(f):=f \circ p_{i}$, for $f: \Gamma / \Gamma_{i} \rightarrow \mathbb{Z}$. Note that $C(X, \mathbb{Z})$ and $\lim _{\longrightarrow}\left(\mathbb{Z}^{\Gamma / \Gamma_{i}}, q_{i}\right)$ are isomorphic as $\Gamma$-modules. Since homology commutes with direct limits, we obtain that

$$
H_{*}(\Gamma, C(X, \mathbb{Z})) \simeq \lim _{\longrightarrow}\left(H_{*}\left(\Gamma, \mathbb{Z}^{\Gamma / \Gamma_{i}}\right), H_{*}\left(q_{i}\right)\right) .
$$

Furthermore, for every $i \geq 1$, Shapiro's lemma gives an isomorphism

$$
\varphi_{i}: H_{*}\left(\Gamma_{i}\right) \rightarrow H_{*}\left(\Gamma, \mathbb{Z}^{\Gamma / \Gamma_{i}}\right) .
$$

We claim that $H_{*}\left(q_{i}\right) \circ \varphi_{i}=\varphi_{i+1} \circ \operatorname{tr}_{\Gamma_{i+1}}^{\Gamma_{i}}$.

Indeed, let $j: \Gamma_{i} \rightarrow \Gamma$ be the inclusion, $\psi: \mathbb{Z} \rightarrow \mathbb{Z}^{\Gamma_{i} / \Gamma_{i+1}}$ be the additive map given by $\psi(1):=\sum_{x \in \Gamma_{i} / \Gamma_{i+1}} \delta_{x}$ and $\theta: \mathbb{Z}^{\Gamma_{i} / \Gamma_{i+1}} \rightarrow \mathbb{Z}^{\Gamma / \Gamma_{i+1}}$ be the additive map given by $\theta\left(\delta_{x}\right)=\delta_{x}$, for $x \in \Gamma_{i} / \Gamma_{i+1}$.

One can readily check that $\varphi_{i+1} \circ \operatorname{tr}_{\Gamma_{i+1}}^{\Gamma_{i}}=(j, \theta)_{*} \circ H_{*}(\psi)=H_{*}\left(q_{i}\right) \circ \varphi_{i}$. This concludes the proof of the proposition.

In particular, for an odometer $\Gamma \curvearrowright X=\lim _{\longleftarrow} \Gamma / \Gamma_{i}$, we have that

$$
H_{0}(\Gamma, C(X, \mathbb{Z})) \simeq\left\{\frac{m}{\left[\Gamma: \Gamma_{i}\right]} \in \mathbb{Q}: m \in \mathbb{Z}, i \geq 1\right\} .
$$


2.4. Ample groupoids. A topological groupoid $G$ is said to be ample if $G$ is locally compact, Hausdorff, étale (in the sense that the range and source maps $r, s: G \rightarrow G$ are local homeomorphisms onto the unit space $\left.G^{(0)}\right)$ and the unit space $G^{(0)}$ is totally disconnected.

Let $G$ be an ample groupoid. The orbit of an $x \in G^{(0)}$ is the set $r\left(s^{-1}(x)\right)$, and the groupoid is said to be minimal if the orbit of each point of $G^{(0)}$ is dense in $G^{(0)}$.

A bisection is a subset $S \subset G$ such that $\left.r\right|_{S}$ and $\left.s\right|_{S}$ are injective. Note that, if $S$ is open, then $\left.r\right|_{S}$ and $\left.s\right|_{S}$ are homeomorphisms onto their images.

Now assume that $G$ is an ample groupoid with compact unit space. The topological full group $[[G]]$ is the group of compact-open bisections $U$ such that $r(U)=$ $s(U)=G^{(0)}$.

There is a homomorphism $\theta$ from $[[G]]$ to the group of homeomorphisms of $G^{(0)}$, given by $\theta_{U}:=r \circ\left(\left.s\right|_{U}\right)^{-1}$. If $G$ is essentially principal (that is, int $\{g \in G: r(g)=$ $\left.s(g)\}=G^{(0)}\right)$, then $\theta$ is injective.

2.5. Homology of ample groupoids. In this sub-section, we recall the definition of the homology groups of an ample groupoid ([15, Definition 2.3], see also the beginning of [10, Section 4]).

Given locally compact, Hausdorff spaces $X$ and $Y$, a local homeomorphism $\pi: X \rightarrow Y$ induces a homomorphism $\pi_{*}: C_{c}(X, \mathbb{Z}) \rightarrow C_{c}(Y, \mathbb{Z})$ given by

$$
\pi_{*}(f)(y):=\sum_{x \in \pi^{-1}(y)} f(x) .
$$

Given an ample groupoid $G$, and $n \geq 1$, let $G^{(n)}$ be the space of sequences $\left(g_{1}, \ldots, g_{n}\right) \in G^{n}$ such that the product $g_{1} \ldots g_{n}$ is well-defined. The topology in $G^{(n)}$ is the one inherited from the product topology in $G^{n}$.

For $i=0, \ldots, n$, let $d_{i}: G^{(n)} \rightarrow G^{(n-1)}$ be given by

$$
d_{i}\left(g_{1}, \ldots, g_{n}\right):= \begin{cases}\left(g_{2}, \ldots, g_{n}\right) & \text { if } i=0 \\ \left(g_{1}, \ldots, g_{i} g_{i+1}, \ldots, g_{n}\right) & \text { if } 1 \leq i \leq n-1 \\ \left(g_{1}, \ldots, g_{n-1}\right) & \text { if } i=n\end{cases}
$$

If $n=1$, let $d_{0}, d_{1}: G^{(1)} \rightarrow G^{(0)}$ be the source and range maps, respectively.

Clearly, the maps $d_{i}$ are local homeomorphisms. Define $\delta_{n}: C_{c}\left(G^{(n)}, \mathbb{Z}\right) \rightarrow$ $C_{c}\left(G^{(n-1)}\right)$ by

$$
\delta_{n}:=\sum_{i=0}^{n}(-1)^{i} d_{i_{*}}
$$

Then

$$
0 \stackrel{\delta_{0}}{\longleftarrow} C_{c}\left(G^{(0)}, \mathbb{Z}\right) \stackrel{\delta_{1}}{\longleftarrow} C_{c}\left(G^{(1)}, \mathbb{Z}\right) \stackrel{\delta_{2}}{\longleftarrow} \ldots
$$

is a chain complex. Denote by $H_{n}(G):=\frac{\operatorname{ker} \delta_{n}}{\operatorname{Im} \delta_{n+1}}$ its homology groups.

Example 2.5. Let $\varphi$ be an action of a group $\Gamma$ on a compact, totally disconnected, Hausdorff space $X$. As a space, the transformation groupoid $G$ associated with $\varphi$ is $G:=\Gamma \times X$ equipped with the product topology. The product of two elements $(h, y),(g, x) \in G$ is defined if and only if $y=g x$, in which case $(h, g x)(g, x):=$ $(h g, x)$. Inversion is given by $(g, x)^{-1}:=\left(g^{-1}, g x\right)$. The unit space $G^{(0)}$ is naturally identified with $X$ and $G$ is ample. Also, $G$ is essentially principal if and only 
if $\varphi$ is topologicall free, and it holds that $C_{r}^{*}(G) \simeq C(X) \rtimes_{r} \Gamma$ and $H_{*}(G) \simeq$ $H_{*}(\Gamma, C(X, \mathbb{Z}))$.

Given $g \in \Gamma$ and $A \subset X$, notice that $s(\{g\} \times A)=A$, and $r(\{g\} \times A)=g A$.

Let us now describe the topological full group $[[G]]$. Take $g_{1}, \ldots, g_{n} \in \Gamma$ and $A_{1}, \ldots, A_{n} \subset X$ clopen sets such that $X=\sqcup_{i=1}^{n} A_{i}=\sqcup_{i=1}^{n} g_{i} A_{i}$ (disjoint unions). Then $U:=\cup_{i=1}^{n}\left\{g_{i}\right\} \times A_{i}$ is a compact-open bisection and $s(U)=r(U)=X$. Therefore, $U \in[[G]]$. Conversely, it is easy to see that any $U \in[[G]]$ is as above.

\section{Counterexamples to the HK conjecture}

In 15, Matui conjectured (HK conjecture) that, given a second countable, étale, minimal, essentially principal groupoid $G$ with unit space homeomorphic to the Cantor set, the following holds:

$$
K_{*}\left(C_{r}^{*}(G)\right) \simeq \bigoplus_{k \geq 0} H_{2 k+*}(G) \text { for } *=0,1 .
$$

As in Example 2.2, let $\Gamma \curvearrowright X=\lim \Gamma / \Gamma_{i}$, with $\Gamma:=\mathbb{Z} \rtimes \mathbb{Z}_{2}$ and, for $i \geq 1, \Gamma_{i}:=$ $n_{i} \mathbb{Z} \rtimes \mathbb{Z}_{2}$, where $\left(n_{i}\right)$ is a strictly increasing sequence of natural numbers such that, for $i \geq 1, n_{i} \mid n_{i+1}$. We are going to compute $K_{*}(C(X) \rtimes \Gamma)$ and $H_{*}(\Gamma, C(X, \mathbb{Z}))$, and conclude that the transformation groupoids associated to these odometers are counterexamples to the HK conjecture.

Note that $\Gamma / \Gamma_{i}$ can be identified with the abelian group $\mathbb{Z}_{n_{i}}=\left\{0, \ldots, n_{i}-1\right\}$. The action of $(1,0) \in \Gamma$ on an element $\left(x_{i}\right) \in \lim \mathbb{Z}_{n_{i}}$ is given by summing 1 in each entry, and the action of $(0,1) \in \Gamma$ is given by multiplying each entry by -1 .

Since $\Gamma \simeq \Gamma_{i}$ for every $i$ and $K_{1}\left(C^{*}\left(\mathbb{Z} \rtimes \mathbb{Z}_{2}\right)\right)=0$ ([1, 10.11.5(a)]), it follows from (22) that $K_{1}(C(X) \rtimes \Gamma)=0$. In fact, $C(X) \rtimes \Gamma$ is an AF algebra (see [2] or [13).

One can also compute $K_{0}(C(X) \rtimes \Gamma)$ by applying (2), but we will instead make use of the following result of Bratteli, Evans and Kishimoto ([2, Theorem 4.1]):

Theorem 3.1. Given an action of $\mathbb{Z} \rtimes \mathbb{Z}_{2}$ on the Cantor set $X$ such that the restricted $\mathbb{Z}$-action is minimal and $(0,1),(1,1) \in \mathbb{Z} \rtimes \mathbb{Z}_{2}$ have at most a finite number $m_{(0,1)}$ and $m_{(1,1)}$ of fixed points, with $m_{(0,1)}+m_{(1,1)}>0$, then $K_{0}\left(C(X) \rtimes \mathbb{Z} \rtimes \mathbb{Z}_{2}\right)$ is isomorphic to

$$
\left(1+(0,1)_{*}\right)\left(\frac{C(X, \mathbb{Z})}{\left(1-(1,0)_{*}\right)(C(X, \mathbb{Z}))}\right) \oplus \mathbb{Z}^{m_{(0,1)}+m_{(1,1)}} .
$$

Actually, the hypothesis that $(0,1)$ or $(1,1)$ must have at least one fixed point does not appear in [2, Theorem 4.1], but Thomsen showed in 21] that the theorem is false without this hypothesis.

In order to apply Theorem 3.1 to the odometers of Example 2.2, we need to compute the number of fixed points of $(0,1),(1,1) \in \mathbb{Z} \rtimes \mathbb{Z}_{2}$ :

Lemma 3.2. Let $\Gamma \curvearrowright \lim \Gamma / \Gamma_{i}$ as in Example 2.2. and denote by $m_{(0,1)}$ and $m_{(1,1)}$ the number of fixed points of $(0,1)$ and $(1,1)$. Then

$$
\begin{aligned}
& m_{(0,1)}= \begin{cases}1 & \text { if } \frac{n_{i+1}}{n_{i}} \text { is even for infinitely many } i \\
1 & \text { if } n_{i} \text { is odd for every } i \\
2 & \text { otherwise, }\end{cases} \\
& m_{(1,1)}= \begin{cases}1 & \text { if } n_{i} \text { is odd for every } i \\
0 & \text { otherwise. }\end{cases}
\end{aligned}
$$


Proof. An element $\left(x_{i}\right) \in \lim \mathbb{Z}_{n_{i}}$ is a fixed point of $(0,1)$ if and only if, for every $i$, $x_{i}=0$ or $x_{i}=\frac{n_{i}}{2}$. Likewise, $\left(x_{i}\right)$ is a fixed point of $(1,1)$ if and only if $x_{i}=\frac{n_{i}+1}{2}$ for every $i$.

Therefore, if $n_{i}$ is odd for every $i$, then the only fixed point of $(0,1)$ is $(0)$ and the only fixed point of $(1,1)$ is $\left(\frac{n_{i}+1}{2}\right)$.

If $\frac{n_{i+1}}{n_{i}}$ is even for infinitely many $i$, then $(1,1)$ admits no fixed points and the only fixed point of $(0,1)$ is $(0)$.

Finally, if there exists $i_{0}$ such that $n_{i_{0}}$ is even, but $\frac{n_{i+1}}{n_{i}}$ is odd for every $i \geq i_{0}$, and $i_{0}$ is minimal with these properties, then the only fixed points of $(0,1)$ are $(0)$ and $\left(0, \ldots, 0, \frac{n_{i_{0}}}{2}, \frac{n_{i_{0}+1}}{2} \ldots\right)$. Furthermore, $(1,1)$ does not admit fixed points.

Proposition 3.3. Let $\Gamma \curvearrowright X=\lim _{\longleftarrow} \Gamma / \Gamma_{i}$ as in Example 2.2. Then $K_{0}(C(X) \rtimes \Gamma) \simeq \begin{cases}\left\{\frac{m}{n_{i}}: m \in \mathbb{Z}, i \geq 1\right\} \oplus \mathbb{Z} & \text { if } \frac{n_{i+1}}{n_{i}} \text { is even for infinitely many } i \\ \left\{\frac{m}{n_{i}}: m \in \mathbb{Z}, i \geq 1\right\} \oplus \mathbb{Z}^{2} & \text { otherwise. }\end{cases}$

Proof. In order to apply Theorem 3.1 let us first compute $\frac{C(X, \mathbb{Z})}{\left(1-(1,0)_{*}\right)(C(X, \mathbb{Z}))}$.

Consider the odometer $\mathbb{Z} \curvearrowright X=\lim _{\longleftarrow} \mathbb{Z}_{n_{i}}$. Notice that $\frac{C(X, \mathbb{Z})}{\left(1-(1,0)_{*}\right)(C(X, \mathbb{Z}))} \simeq$ $H_{0}(\mathbb{Z}, C(X, \mathbb{Z}))$. Therefore, by (4),

$$
\frac{C(X, \mathbb{Z})}{\left(1-(1,0)_{*}\right)(C(X, \mathbb{Z}))} \simeq\left\{\frac{m}{n_{i}}: m \in \mathbb{Z}, i \geq 1\right\} .
$$

Given $j \geq 1$ and $k \in \mathbb{Z}_{n_{j}}$, let $U(j, k):=\left\{\left(x_{i}\right) \in X: x_{j}=k\right\}$. Observe that $\left((1,0)_{*}\right)^{k}\left(1_{U(j, 0)}\right)=1_{U(j, k)}$, and $(0,1)_{*}\left(1_{U(j, k)}\right)=1_{U(j,-k)}$. From these facts, it follows that $(0,1)_{*}$ acts trivially on $\frac{C(X, \mathbb{Z})}{\left(1-(1,0)_{*}\right)(C(X, \mathbb{Z}))}$.

The result now is a consequence of Theorem 3.1 and Lemma 3.2 .

The following lemma will be useful for computing the homology of the $\left(\mathbb{Z} \rtimes \mathbb{Z}_{2}\right)$ odometers that we are investigating in this section.

Lemma 3.4. Let $\sigma$ be an involutive homeomorphism on a compact, totally disconnected, Hausdorff space $Y$ such that $F_{\sigma}:=\{y \in Y: \sigma(y)=y\}$ is finite. Then, for $k \geq 0, H_{2 k+1}\left(\mathbb{Z}_{2}, C(Y, \mathbb{Z})\right)=\left(\mathbb{Z}_{2}\right)^{F_{\sigma}}$.

Proof. By [22, Theorem 6.2.2], we have that

$$
H_{2 k+1}\left(\mathbb{Z}_{2}, C(Y, \mathbb{Z})\right)=\frac{\{f \in C(Y, \mathbb{Z}): f \circ \sigma=f\}}{\{f+f \circ \sigma: f \in C(Y, \mathbb{Z})\}} .
$$

Let $E: \frac{\{f \in C(Y, \mathbb{Z}): f \circ \sigma=f\}}{\{f+f \circ \sigma: f \in C(Y, \mathbb{Z})\}} \rightarrow\left(\mathbb{Z}_{2}\right)^{F_{\sigma}}$ be given by evaluation at the points of $F_{\sigma}$. Clearly, $E$ is a well-defined homomorphism, and we will show that it is bijective.

For each $y \in F_{\sigma}$, take $A_{y} \subset Y$ clopen set such that $A_{y} \cap F_{\sigma}=\{y\}$, and $\sigma\left(A_{y}\right)=A_{y}$. Note that $E\left(\left[1_{A_{y}}\right]\right)=\delta_{y}$, for every $y \in F_{\sigma}$. Hence, $E$ is surjective.

Let us now verify injectivity of $E$. Take $f \in C(Y, \mathbb{Z})$ such that $f \circ \sigma=f$ and $E([f])=0$. We will show that $[f]=0$. Clearly, we can assume that $\left.f\right|_{F_{\sigma}}=0$. Then $f$ can be written as a linear combination of functions of the form $1_{B}+1_{\sigma(B)}$, for certain $B \subset Y$ clopen sets such that $B \cap \sigma(B)=\emptyset$. This concludes the proof of the lemma. 
Theorem 3.5. Let $\Gamma \curvearrowright X=\lim _{\longleftarrow} \Gamma / \Gamma_{i}$ as in Example 2.2. Then, for $k \geq 1$,

$$
\begin{aligned}
H_{0}(\Gamma, C(X, \mathbb{Z})) & =\left\{\frac{m}{n_{i}}: m \in \mathbb{Z}, i \geq 1\right\} \\
H_{2 k}(\Gamma, C(X, \mathbb{Z})) & =0, \\
H_{2 k-1}(\Gamma, C(X, \mathbb{Z})) & = \begin{cases}\mathbb{Z}_{2} & \text { if } \frac{n_{i+1}}{n_{i}} \text { is even for infinitely many } i \\
\left(\mathbb{Z}_{2}\right)^{2} & \text { otherwise. }\end{cases}
\end{aligned}
$$

Proof. As $\mathbb{Z} \rtimes \mathbb{Z}_{2} \simeq \mathbb{Z}_{2} * \mathbb{Z}_{2}$, it follows from [22, Corollary 6.2.10] and Proposition 2.4 that, for $k \geq 1, H_{2 k}(\Gamma, C(X, \mathbb{Z}))=0$. Moreover, $H_{0}(\Gamma, C(X, \mathbb{Z}))=\left\{\frac{m}{n_{i}}: m \in\right.$ $\mathbb{Z}, i \geq 1\}$ (see (4) ).

Furthermore, from Lemmas 3.2 and 3.4 it follows that, for $k \geq 1$,

$$
H_{2 k+1}(\Gamma, C(X, \mathbb{Z}))=\left(\mathbb{Z}_{2}\right)^{r},
$$

where $r=1$ if $\frac{n_{i+1}}{n_{i}}$ is even for infinitely many $i$, and $r=2$ otherwise.

Finally, we compute $H_{1}(\Gamma, C(X, \mathbb{Z}))$. For every $i,\left(\Gamma_{i}\right)_{\mathrm{ab}} \simeq \mathbb{Z}_{2} \times \mathbb{Z}_{2}$ with canonical generators $\left(n_{i}, 0\right) \Gamma_{i}^{\prime}$ and $(0,1) \Gamma_{i}^{\prime}$. Under these identifications, and using (3), it is easy to see that $\operatorname{tr}_{\Gamma_{i+1}}^{\Gamma_{i}}: \mathbb{Z}_{2} \times \mathbb{Z}_{2} \rightarrow \mathbb{Z}_{2} \times \mathbb{Z}_{2}$ is given by $\operatorname{tr}_{\Gamma_{i+1}}^{\Gamma_{i}}(1,0)=(1,0)$ and

$$
\operatorname{tr}_{\Gamma_{i+1}}^{\Gamma_{i}}(0,1)= \begin{cases}(1,0) & \text { if } \frac{n_{i+1}}{n_{i}} \text { is even } \\ (0,1) & \text { otherwise. }\end{cases}
$$

It follows from Proposition 2.4 that $H_{1}(\Gamma, C(X, \mathbb{Z}))=\left(\mathbb{Z}_{2}\right)^{r}$, where $r=1$ if $\frac{n_{i+1}}{n_{i}}$ is even for infinitely many $i$, and $r=2$ otherwise.

\section{On the AH CONJECture For odometers}

Let $G$ be a second countable, ample, essentially principal and minimal groupoid with unit space homeomorphic to the Cantor set. Matui conjectured in [15] ( $A H$ conjecture) that there is an exact sequence

$$
H_{0}(G) \otimes \mathbb{Z}_{2} \stackrel{j}{\rightarrow}[[G]]_{\mathrm{ab}} \stackrel{I}{\rightarrow} H_{1}(G) \rightarrow 0 .
$$

The map $I:[[G]]_{\mathrm{ab}} \rightarrow H_{1}(G)$ is given by $I\left(U[[G]]^{\prime}\right):=\left[1_{U}\right]$, for $U \in[[G]]$ (this is defined for any ample groupoid with compact unit space).

Let us describe $j: H_{0}(G) \otimes \mathbb{Z}_{2} \rightarrow[[G]]_{\text {ab }}$, for $G$ an ample groupoid with compact unit space such that the orbit of each point of $G^{(0)}$ has at least three points. Given $F \subset G$ compact-open bisection such that $s(F) \cap r(F)=\emptyset$, notice that $\tau_{F}:=$ $F \cup F^{-1} \cup\left(G^{(0)} \backslash(s(F) \cup r(F))\right) \in[[G]]$. The map $j$ is the unique homomorphism such that $j\left(\left[1_{s(F)}\right] \otimes 1\right)=\tau_{F}[[G]]^{\prime}$ for each such $F$. See [16, Theorem 7.2] for a proof that $j$ is well-defined. It is easy to see that $I \circ j=0$.

Given a set $X$, we denote by $S_{X}$ the group of bijections on $X$.

The proof of the following lemma uses a technique employed in [14, Proposition 2.1] and [6, Proposition 4.6].

Lemma 4.1. Let $\Lambda$ be a finite index subgroup of $\Gamma$ and $G$ the transformation groupoid associated with $\Gamma \curvearrowright \Gamma / \Lambda$. Then $[[G]] \simeq \Lambda^{\Gamma / \Lambda} \rtimes S_{\Gamma / \Lambda}$ and $[[G]]_{\mathrm{ab}} \simeq$ $\Lambda_{\mathrm{ab}} \times\left(S_{\Gamma / \Lambda}\right)_{\mathrm{ab}}$. 
Proof. There is an epimorphism $\theta:[[G]] \rightarrow S_{\Gamma / \Lambda}$ given by $\theta_{U}:=r \circ\left(\left.s\right|_{U}\right)^{-1}$, for $U \in[[G]]$. Fix representatives $g_{1}, \ldots, g_{n}$ for $\Gamma / \Lambda$ and define $\eta: S_{\Gamma / \Lambda} \rightarrow[[G]]$ by $\eta(\sigma):=\cup_{i=1}^{n}\left\{\left(g_{\sigma(i)} g_{i}^{-1}, g_{i} \Lambda\right)\right\}$, for $\sigma \in S_{\Gamma / \Lambda}$. Note that $\eta$ is a homomorphism which is a right inverse for $\theta$. In particular, $[[G]] \simeq \operatorname{ker} \theta \rtimes S_{\Gamma / \Lambda}$. Clearly,

$$
\operatorname{ker} \theta \simeq g_{1} \Lambda g_{1}^{-1} \times \cdots \times g_{n} \Lambda g_{n}^{-1} \simeq \Lambda^{\Gamma / \Lambda} \text {. }
$$

Therefore, there is an isomorphism from $\Lambda_{\mathrm{ab}} \times\left(S_{\Gamma / \Lambda}\right)_{\mathrm{ab}}$ into $[[G]]_{\mathrm{ab}}$ induced by the embeddings $\eta: S_{\Gamma / \Lambda} \rightarrow[[G]]$, defined in the previous paragraph, and $\zeta: \Lambda \rightarrow$ $[[G]]$ given by $\zeta(\lambda):=\{(\lambda, \Lambda)\} \cup\left(\{e\} \times\{\Lambda\}^{c}\right)$, for $\lambda \in \Lambda$.

Lemma 4.2. Let $\Lambda$ be a finite index subgroup of $\Gamma$ such that $[\Gamma: \Lambda] \geq 3$, and $G$ the transformation groupoid associated with $\Gamma \curvearrowright \Gamma / \Lambda$. Then the following sequence is split exact:

$$
0 \rightarrow H_{0}(G) \otimes \mathbb{Z}_{2} \stackrel{j}{\rightarrow}[[G]]_{\mathrm{ab}} \stackrel{I}{\rightarrow} H_{1}(G) \rightarrow 0 .
$$

Proof. Identify $[[G]]_{\mathrm{ab}}$ with $\Lambda_{\mathrm{ab}} \times\left(S_{\Gamma / \Lambda}\right)_{\mathrm{ab}}$ as in the previous lemma. Note that $\left(S_{\Gamma / \Lambda}\right)_{\mathrm{ab}} \simeq \mathbb{Z}_{2}$ and, by Shapiro's lemma, $H_{1}(G) \simeq \Lambda_{\mathrm{ab}}$ and $H_{0}(G) \otimes \mathbb{Z}_{2} \simeq \mathbb{Z}_{2}$. Under these identifications, $I: \Lambda_{\mathrm{ab}} \times \mathbb{Z}_{2} \rightarrow \Lambda_{\mathrm{ab}}$ is the canonical projection, and $j: \mathbb{Z}_{2} \rightarrow \Lambda_{\mathrm{ab}} \times \mathbb{Z}_{2}$ is the canonical inclusion.

Theorem 4.3. Let $G$ be the transformation groupoid associated to an odometer $\Gamma \curvearrowright \lim \Gamma / \Gamma_{i}$. The following sequence is exact.

$$
0 \rightarrow H_{0}(G) \otimes \mathbb{Z}_{2} \stackrel{j}{\rightarrow}[[G]]_{\mathrm{ab}} \stackrel{I}{\rightarrow} H_{1}(G) \rightarrow 0 .
$$

Proof. Given $i \in \mathbb{N}$, let $G_{i}$ be the transformation groupoid associated to $\Gamma \curvearrowright \Gamma / \Gamma_{i}$. The result follows from the fact that $H_{*}(G)=\varliminf_{\longrightarrow} H_{*}\left(G_{i}\right),[[G]]_{\mathrm{ab}}=\lim _{\longrightarrow}\left[\left[G_{i}\right]\right]_{\mathrm{ab}}$ and Lemma 4.2

Note that, given an odometer $\Gamma \curvearrowright X=\lim \Gamma / \Gamma_{i}$, it holds that

$$
H_{0}(\Gamma, C(X, \mathbb{Z})) \otimes \mathbb{Z}_{2} \simeq \begin{cases}0 & \text { if }\left[\Gamma_{i}: \Gamma_{i+1}\right] \text { is even for infinitely many } i \\ \mathbb{Z}_{2} & \text { otherwise. }\end{cases}
$$

We do not know whether there is an odometer for which the exact sequence (5) does not split.

\section{REFERENCES}

[1] Blackadar, B. K-theory for operator algebras, vol. 5 of Mathematical Sciences Research Institute Publications. Springer-Verlag, New York, 1986. 11 6

[2] Bratteli, O., Evans, D. E., and Kishimoto, A. Crossed products of totally disconnected spaces by $Z_{2} * Z_{2}$. Ergodic Theory Dynam. Systems 13, 3 (1993), 445-484. 6]

[3] Brown, K. S. Cohomology of groups, vol. 87 of Graduate Texts in Mathematics. SpringerVerlag, New York, 1994. Corrected reprint of the 1982 original.4

[4] Brownlowe, N., Mundey, A., Pask, D., Spielberg, J., and Thomas, A. $C^{*}$-algebras associated to graphs of groups. Adv. Math. 316 (2017), 114-186. 3

[5] CARrión, J. R. Classification of a class of crossed product $C^{*}$-algebras associated with residually finite groups. J. Funct. Anal. 260, 9 (2011), 2815-2825. 3

[6] Contez, M. A. I., And Medynets, K. Orbit equivalence rigidity of equicontinuous systems. J. Lond. Math. Soc. (2) 94, 2 (2016), 545-556. 1 28 
[7] Cortez, M. I., And Petite, S. G-odometers and their almost one-to-one extensions. J. Lond. Math. Soc. (2) 78, 1 (2008), 1-20. 3

[8] DE Cornulier, Y. Groupes pleins-topologiques (d'après Matui, Juschenko, Monod, ...). Astérisque, 361 (2014), Exp. No. 1064, viii, 183-223. 2

[9] Dyer, J., Hurder, S., and Lukina, O. The discriminant invariant of Cantor group actions. Topology Appl. 208 (2016), 64-92. 10

[10] Farsi, C., Kumjian, A., Pask, D., And Sims, A. Ample groupoids: equivalence, homology, and Matui's HK conjecture. arXiv preprint arXiv:1808.07807 (2018). 15

[11] Green, P. The structure of imprimitivity algebras. J. Funct. Anal. 36, 1 (1980), 88-104. 3

[12] IoAna, A. Cocycle superrigidity for profinite actions of property (T) groups. Duke Math. J. 157, 2 (2011), 337-367. 1

[13] Kumjian, A. An involutive automorphism of the Bunce-Deddens algebra. C. R. Math. Rep. Acad. Sci. Canada 10, 5 (1988), 217-218. 10

[14] Matui, H. Some remarks on topological full groups of Cantor minimal systems II. Ergodic Theory Dynam. Systems 33, 5 (2013), 1542-1549. 8

[15] Matui, H. Étale groupoids arising from products of shifts of finite type. Adv. Math. 303 (2016), 502-548. 156

[16] Nekrashevych, V. Simple groups of dynamical origin. Ergodic Theory and Dynamical Systems (2017), 1-26. 8

[17] NekrasheVyCh, V. Palindromic subshifts and simple periodic groups of intermediate growth. Ann. of Math. (2) 187, 3 (2018), 667-719. 1

[18] Orfanos, S. Generalized Bunce-Deddens algebras. Proc. Amer. Math. Soc. 138, 1 (2010), 299-308. [1

[19] Ortega, E. Homology of the Katsura-Exel-Pardo groupoid. arXiv preprint arXiv:1806.09297 (2018). 1

[20] Scarparo, E. On the $C^{*}$-algebra generated by the Koopman representation of a topological full group. arXiv preprint arXiv:1705.07665 (2017). 1

[21] Thomsen, K. The homoclinic and heteroclinic $C^{*}$-algebras of a generalized one-dimensional solenoid. Ergodic Theory Dynam. Systems 30, 1 (2010), 263-308. 6

[22] Weibel, C. A. An introduction to homological algebra, vol. 38 of Cambridge Studies in Advanced Mathematics. Cambridge University Press, Cambridge, 1994. 78

Departamento de Matemática, Universidade Federal de Santa Catarina, 88040-970 FLORIANÓPOLIS-SC, BRAZIL

E-mail address: duduscarparo@gmail.com 